\title{
Fock quantization of a scalar field with time dependent mass on the three-sphere: unitarity and uniqueness
}

\author{
Jerónimo Cortez, $1, *$ Guillermo A. Mena Marugán, $2,+$ and José M. Velhinho3, \\ ${ }^{1}$ Departamento de Física, Facultad de Ciencias, \\ Universidad Nacional Autónoma de México, México D.F. 04510, Mexico. \\ ${ }^{2}$ Instituto de Estructura de la Materia, \\ CSIC, Serrano 121, 28006 Madrid, Spain. \\ ${ }^{3}$ Departamento de Física, Universidade da Beira Interior, \\ R. Marquês D'Ávila e Bolama, 6201-001 Covilhã, Portugal.
}

We study the Fock description of a quantum free field on the three-sphere with a mass that depends explicitly on time, also interpretable as an explicitly time dependent quadratic potential. We show that, under quite mild restrictions on the time dependence of the mass, the specific Fock representation of the canonical commutation relations which is naturally associated with a massless free field provides a unitary dynamics even when the time varying mass is present. Moreover, we demonstrate that this Fock representation is the only acceptable one, up to unitary equivalence, if the vacuum has to be $S O(4)$-invariant (i.e., invariant under the symmetries of the field equation) and the dynamics is required to be unitary. In particular, the analysis and uniqueness of the quantization can be applied to the treatment of cosmological perturbations around Friedmann-Robertson-Walker spacetimes with the spatial topology of the three-sphere, like e.g. for gravitational waves (tensor perturbations). In addition, we analyze the extension of our results to free fields with a time dependent mass defined on other compact spatial manifolds. We prove the uniqueness of the Fock representation in the case of a two-sphere as well, and discuss the case of a three-torus.

PACS numbers: 04.62.+v, 04.60.-m, 98.80.Qc, 03.70.+k 


\section{INTRODUCTION}

The quantization of a classical system is not a uniquely determined procedure. There exist ambiguities in different steps of the process which generally lead to inequivalent quantum theories and, hence, to different quantum physical descriptions. In standard quantum mechanics, for systems with a finite number of degrees of freedom, these ambiguities are usually resolved by adopting a strongly continuous, irreducible, and unitary representation of the Weyl algebra associated with the linear phase space. The Stonevon Neumann theorem [1] guarantees the uniqueness of the representation up to unitary equivalence, which does not affect the physical content of the so-constructed quantum theory. However, for infinite dimensional systems, an analog theorem does not exist in generic situations. For instance, for linear quantum fields and assuming that it is possible to adopt a Fock quantization, where a concept of particle is available, it is well known that there exists an infinite number of inequivalent Fock representations of the Weyl relations [2]. In the case of Klein-Gordon fields in Minkowski spacetime, the symmetry of the background spacetime is so large that it provides a criterion to select a unique representation, modulo unitary equivalence. Namely, one adopts the unique Fock representation based on a Poincaré invariant physical state, which can viewed as the vacuum. Poincaré invariance suffices to select an essentially unique complex structure, which is the mathematical structure which encodes the physical freedom existing in the Fock quantization (see e.g. Ref. [3] for a discussion). For less symmetric background spacetimes, one cannot appeal to Poincaré invariance in order to arrive at a unique Fock representation. Even so, provided that the spacetime is at least stationary, one can still select a preferred complex structure for a Klein-Gordon field by imposing some suitable energy criterion [4].

For generic curved spacetimes or for linear scalar fields with a time dependent mass (or, equivalently, subject to a time dependent quadratic potential), the stationarity is

\footnotetext{
*Electronic address: jacq@fciencias.unam.mx

$\dagger$ Electronic address: mena@iem.cfmac.csic.es

${ }^{\ddagger}$ Electronic address: jvelhi@ubi.pt
} 
lost. Then, in general, no uniqueness criterion exists and many unitarily inequivalent Fock representations are possible, so that the quantum field physics is not uniquely determined. In spite of this, some uniqueness theorems have been obtained recently for linear fields in the context of quantum cosmology [5- 8] invoking not only the symmetries of the field equation, which are severely restricted for general curved spacetimes, but also the unitarity of the quantum field dynamics. In more detail, the uniqueness of the Fock representation has been proved for a linear free field with time dependent mass defined on the circle [5, 6], or on the two-sphere under the requirement of axisymmetry [7, 8].

Apart from the academical interest of these examples of uniqueness theorems, their physical interest comes from the symmetry reduction of general relativity by two spacelike Killing vectors for compact spatial topologies. This symmetry reduction leads to a family of cosmological models with inhomogeneities which was first studied by Gowdy [9]. Gowdy classified the possible compact topologies, demonstrating that the spatial sections of the spacetime must be homeomorphic to either the three-torus $T^{3}$, the three-sphere $S^{3}$, or the three-handle $S^{2} \times S^{1}$. The Gowdy cosmologies can be interpreted as a compact homogeneous Bianchi universe containing gravitational waves. In the case of linearly polarized waves, the infinite number of degrees of freedom present in the inhomogeneities can be described in terms of a scalar field which satisfies a wave equation with a time dependent mass term [7]. For Gowdy cosmologies with the topology of a three-torus, the wave equation is that corresponding to a static $(1+1)$-dimensional spacetime whose spatial manifold is a circle (equipped with the standard metric) [5, 10]. For the rest of possible topologies in the Gowdy models, the wave equation corresponds to a static $(1+2)$ dimensional spacetime, the spatial manifold being a two-sphere [8, 11]. In this later case, in addition, the field which describes the gravitational waves has to be axisymmetric.

The Fock representation selected by the criteria of invariance of the vacuum under the symmetries of the field equation and unitarity of the dynamics is the one which is naturally associated with a free massless scalar field. In the proof of these uniqueness theorems, a fundamental role is played by the compactness of the spatial sections of the spacetime in which the field propagates. A natural question is whether these uniqueness 
results can be extended to linear fields with time dependent mass propagating in more realistic spacetimes, maintaining the assumption of compact spatial topology. In particular, one is interested in $(1+3)$-dimensional spacetimes, corresponding to the actually observed dimensionality. This would allow the application of the results to physically relevant scenarios without the recourse to a lower dimensionality motivated from symmetry reductions of general relativity.

In this work, we will focus our discussion on a linear field with time dependent mass defined on $S^{3}$. The interest of this case is manifold. Obviously, the analysis has a straightforward application for the quantization of test fields on a Friedmann-Robertson-Walker (FRW) background with the spatial topology of a three-sphere. For a Klein-Gordon scalar field, e.g., a rescaling by the scale factor of the FRW universe transforms the field equation to the form of a linear wave equation with time dependent mass in a static $(1+3)$-spacetime whose spatial manifold is $S^{3}$ (with the standard metric). Besides, in a suitable Lorentz gauge, the sourceless Maxwell equations on a FRW background with $S^{3}$ spatial topology lead to field equations for the vector potential that are of the considered form (i.e., wave equations for the propagation in the sphere) with a vanishing mass [12].

But the framework in which the discussion finds probably the most natural application is in the treatment of perturbations of FRW spacetimes. The study of perturbations around classical FRW cosmologies at first order (i.e., keeping in the field equations only linear terms in the perturbations) was originally developed by Lifshitz [13]. An attempt to provide a description of the perturbations free of the physical ambiguities introduced by gauge freedom was made by Hawking [14]. Later, Olson obtained a covariant formulation of the perturbative equations for the case of an isentropic perfect fluid in a background with vanishing spatial curvature [15]. A truly gauge invariant formalism for the analysis of generic cosmological perturbations was constructed by Bardeen [16, 17]. Without trying to be exhaustive in the literature about cosmological perturbations, other aspects of linear perturbations of FRW spacetimes can be found in Refs. [18, 19], specially for the case of a scalar field with arbitrary potential in a flat FRW background.

In particular, for isotropic perturbations of the energy-momentum tensor (like e.g. for a 
perfect fluid) which are besides adiabatic, the gauge invariant energy density perturbation amplitude, or equivalently the (only independent) Bardeen potential, satisfies a second order linear differential equation which, after a rescaling by a suitable time function, can be written as a wave equation with a time dependent mass term [16, 18]. This wave equation is that of a static and homogenous $(1+3)$-spacetime with the same spatial topology as the FRW background. In the case of a FRW universe whose spatial sections are three-spheres, one arrives to a wave equation for a field on $S^{3}$, precisely the case that we will consider in detail in this work. Furthermore, the tensor perturbations of a FRW spacetime, describing the content of gravitational waves, turn out to satisfy as well a field equation of the considered form if the perturbations of the energy-momentum tensor are isotropic: after a rescaling of their amplitudes by the FRW scale factor (and adopting conformal time), one arrives at a wave equation for the tensor perturbations which includes a time dependent mass [16]. Again, when the spatial topology of the FRW universe is a three-sphere, these perturbations are given by a tensor field on $S^{3}$.

For the case of a scalar field with nonvanishing (constant) mass as the matter content, a detailed study of the perturbations of a FRW spacetime with the spatial topology of $S^{3}$ was carried out by Halliwell and Hawking in the context of quantum cosmology [20]. From that work one can obtain (apart from the results about tensor perturbations commented above) the equation satisfied by the perturbations of the matter field expanded in harmonics on the three-sphere [12], in a gauge where the perturbations of the spatial metric contain no tensor harmonics derived from the scalar ones [20]. After rescaling the matter field perturbations by the FRW scale factor, a careful analysis of the resulting equation in conformal time shows that the solutions for large harmonic number $n$ (namely, for large negative eigenvalue of the Laplace-Beltrami operator on $S^{3}$ ) reproduce in fact those for a free field on $S^{3}$ in the presence of a time dependent mass term, up to asymptotic corrections which do not affect the discussion presented in the rest of this work.

Then, the uniqueness that we will prove for the Fock representation of a linear scalar field with time varying mass implies that, in all the examples commented above (and after adopting the rescaling of the field indicated to arrive at the studied equation), one can 
select a preferred Fock quantization, picked up by the symmetries of the field equation and the unitary implementation of the dynamics. Furthermore, we will discuss possible extensions of our uniqueness result for compact spatial manifolds other than the threesphere. In particular, we will demonstrate that the uniqueness is reached as well for a field on the two-sphere, without any need to impose axisymmetry (therefore generalizing the results of Ref. [7]). In addition, we will comment on the case of a three-torus, which finds application in the treatment of perturbations of a FRW spacetime with a flat and compact spatial topology, according to our comments above.

The rest of the paper is organized as follows. Sec. 2 contains some basic considerations about fields on $S^{3}$ and their quantization with Fock techniques. We then show in Sec. 3 that the Fock representation associated with a free massless field provides a unitary implementation of the dynamics even if a time dependent mass term is present in the field equation (under quite mild conditions on the time dependence of the mass). Sec. 4 is devoted to prove that, if one restricts oneself to complex structures with the invariance of the field equation and requires a unitary dynamics, all possible Fock representations are unitarily equivalent. In this sense, the quantization is unique. The generalization of the unitarity and uniqueness results for fields defined on compact spatial manifolds other than $S^{3}$ is discussed in Sec. 5, where we also present the conclusions. Finally, an appendix is added which contains some calculations needed in the proof of uniqueness.

\section{QUANTUM SCALAR FIELD ON $S^{3}$}

\section{A. The field and its decomposition in harmonics}

Let us consider a scalar field $\phi$ propagating in a globally hyperbolic, static $(1+3)$ -

background whose spatial manifold is the three-sphere $S^{3}$ equipped with the standard metric, i.e., the metric induced from the Euclidean metric on $E^{4}$ :

$$
h_{a b}=\mathrm{d} \chi_{a} \otimes \mathrm{d} \chi_{b}+\sin ^{2}(\chi) \mathrm{d} \theta_{a} \otimes \mathrm{d} \theta_{b}+\sin ^{2}(\chi) \sin ^{2}(\theta) \mathrm{d} \sigma_{a} \otimes \mathrm{d} \sigma_{b} .
$$

Here, $\sigma \in S^{1}$ and the rest of coordinates have a range of $\pi$. The $(1+3)$-dimensional 
spacetime has the topology of $\mathbb{I} \times S^{3}$, where $\mathbb{I}$ is an interval of the real line, and its metric is $g_{a b}=-\mathrm{d} t_{a} \mathrm{~d} t_{b}+h_{a b}$.

The scalar field satisfies a linear wave equation which includes a time dependent mass term, namely, a potential of the form $V(\phi)=f(t) \phi^{2} / 2$ :

$$
\ddot{\phi}-\Delta \phi+f(t) \phi=0
$$

where $\Delta$ denotes the Laplace-Beltrami operator on $S^{3}, f(t)$ is a real function on $\mathbb{I}$, and the dot stands for the time derivative.

The canonical phase space $\Gamma$ is the space of Cauchy data at some fixed time $t_{0}$, $\{(\varphi, P)\}=\left\{\left(\phi_{\mid t_{0}}, \sqrt{h} \dot{\phi}_{\mid t_{0}}\right)\right\}$ where $h=\sin ^{2}(\theta) \sin ^{4}(\chi)$ is the determinant of the metric on the spatial section $S^{3}$. The symplectic structure (independent of the choice of time section) is

$$
\Omega\left[\left(\varphi_{1}, P_{1}\right),\left(\varphi_{2}, P_{2}\right)\right]=\oint d^{3} x\left(\varphi_{2} P_{1}-\varphi_{1} P_{2}\right) .
$$

The corresponding nonzero Poisson brackets are $\left\{\varphi(x), P\left(x^{\prime}\right)\right\}=\delta\left(x-x^{\prime}\right)$, where $\delta(x)$ is the Dirac delta on $S^{3}$, and $x$ collectively denotes the (hyper)spherical coordinates on $S^{3}$, $x:=(\chi, \theta, \sigma)$.

It is clear from the field equation (2) that the group of rotations $S O(4)$ is a group of symmetries of the field dynamics, since the metric, and therefore the Laplacian, is $S O(4)$-invariant. Upon quantization, we will therefore look for a unitary implementation not just of the field dynamics, but of the group of symmetries $S O(4)$ as well.

The most natural way to obtain a unitary implementation of a group of symmetries, or more generally of any set of symplectic transformations, is to define the quantum representation of the canonical commutation relations (CCR's) by means of a state of the Weyl algebra (interpretable as a vacuum) which is invariant under the transformations in question. In the case of $S O(4)$, a representation which is well known to be invariant is the massless free field representation, since it is defined by a complex structure in phase space which is determined exclusively by the metric and the Laplacian.

As we will see, the dynamics of our field turns out to be implemented also unitarily in the free field representation for any (sufficiently regular) function $f(t)$ in the field equation (2). 
In order to discuss these issues in more detail, it is convenient to adopt a description of the field in terms of harmonics, i.e., solutions of the eigenvalue equation for the Laplace-Beltrami operator [21]. As it is well known, fields on $S^{3}$ admit a decomposition in (hyper)spherical harmonics [12, 13, 20, 22], just like in the more familiar $S^{2}$ case. Thus, the scalar field $\phi(t, x)$ can be written as a linear combination of modes $Q_{n \ell m}(x)$, which are eigenfunctions of the Laplace-Beltrami operator $\Delta$ with eigenvalue $-n(n+2)$, where $n=0,1,2, \ldots$ Explicitly,

$$
\phi(t, x)=\sum_{n, \ell, m} A_{n \ell m}(t) Q_{n \ell m}(x),
$$

where the coefficients $A_{n \ell m}$ are functions of time and $\ell$ and $m$ take the values $\ell=0, \ldots, n$ and $m=-\ell, \ldots, \ell$, respectively. The scalar harmonics $Q_{n \ell m}$, normalized with respect to the volume element on $S^{3}$, have the form

$$
Q_{n \ell m}(\chi, \theta, \sigma)=2^{\ell+\frac{1}{2}}(\ell !) \sqrt{\frac{(n-\ell) !(n+1)}{\pi(n+\ell+1) !}} \sin ^{\ell}(\chi) C_{n-\ell}^{(\ell+1)}[\cos (\chi)] Y_{\ell m}(\theta, \sigma),
$$

where $Y_{\ell m}$ are the usual spherical harmonics on $S^{2}$ and $C_{n-\ell}^{(\ell+1)}[\cos (\chi)]$ denote the Gegenbauer polynomials [23, 24]. With fixed $n$, the functions $Q_{n \ell m}$ span an irreducible representation of $S O(4)$ of dimension $(n+1)^{2}$ (see e.g. Ref. [12]).

Let us restrict our discussion to the particular case of a real field. We start by writing down an alternative expression for the decomposition (4) of a (generally complex) field $\phi$ which is better adapted to the real case,

$$
\phi=\sum_{n, \ell} q_{n \ell 0} Q_{n \ell 0}+\sqrt{2} \sum_{n, \ell, m>0} q_{n \ell m} \Re\left[Q_{n \ell m}\right]+\sqrt{2} \sum_{n, \ell, m>0} q_{n \ell-m} \Im\left[Q_{n \ell m}\right] .
$$

Here, the integer $m$ is strictly positive in the last two sums, and the symbols $\Re$ and $\Im$ denote, respectively, the real and imaginary parts. We note first that the well known behavior of the spherical harmonics under complex conjugation,

$$
Y_{\ell m}^{*}=(-1)^{m} Y_{\ell-m}
$$

implies a similar behavior for the scalar harmonics on $S^{3}$,

$$
Q_{n \ell m}^{*}=(-1)^{m} Q_{n \ell-m}
$$


because the remaining factors in $Q_{n \ell m}$ are real and independent of $m$ [see Eq. (5)]. In addition, the functions $Q_{n \ell 0}$ are real as well. Therefore a general real field is obtained just by restricting all considerations to real coefficients in the decomposition (66).

Thus, the configuration space of a real scalar field in $S^{3}$ is in one-to-one correspondence with the space of all real coefficients

$$
\left\{\left(q_{n \ell m}\right) ; n=0,1, \ldots ; \ell=0, \ldots, n ; m=-\ell, \ldots, \ell\right\}
$$

Taking into account the field equation (2), the orthogonality properties of the harmonics $Q_{n \ell m}$, and relation (8), it is straightforward to see that these modes obey completely decoupled equations of motion, which moreover depend only on $n$. In particular, all the modes $q_{n \ell m}$ with the same $n$ satisfy the same equation of motion:

$$
\ddot{q}_{n \ell m}+\left(\omega_{n}^{2}+f\right) q_{n \ell m}=0
$$

where

$$
\omega_{n}^{2}:=n(n+2)
$$

Note that, if we call $k:=n+1$, we have $\omega_{k}^{2}=k^{2}-1$ with the above definition. Actually, we might absorb the contribution of -1 in $\omega_{k}^{2}$ by redefining $f(t)$, and then work in the unit mass representation, with $\omega_{k}=k$. Since this will obscure the interpretation of the quantities appearing in our discussion, we will continue to use the mode number $n$ as our label and the original function $f$ in our equations.

So, as we have seen, the modes $q_{n \ell m}$ describe completely decoupled degrees of freedom. We will call $\mathcal{Q}_{n}$ the corresponding subset of the full configuration space associated with a fixed $n$, i.e., $\mathcal{Q}_{n}$ is the linear space of dimension $(n+1)^{2}$ spanned by the modes $q_{n \ell m}$ with the same label $n$ (while $\ell \in\{0, \ldots, n\}$ and in turn $m \in\{-\ell, \ldots, \ell\}$ ).

The momentum variables canonically conjugate to the above configuration variables are $p_{n \ell m}=\dot{q}_{n \ell m}$. From the basic Poisson bracket between $\varphi$ and $P$ and the orthogonality of the scalar harmonics, one can check that $q_{n \ell m}$ and the introduced $p_{n \ell m}$ form a complete set of variables in phase space which is canonical, i.e.,

$$
\left\{q_{n \ell m}, p_{n^{\prime} \ell^{\prime} m^{\prime}}\right\}=\delta_{n n^{\prime}} \delta_{\ell \ell^{\prime}} \delta_{m m^{\prime}}
$$


with all other Poisson brackets between this set of variables being equal to zero.

Clearly, each space $\mathcal{Q}_{n}$, as well as the corresponding momentum space $\mathcal{P}_{n}$, carries an irreducible representation of $S O(4)$ of dimension $(n+1)^{2}$. Moreover, the representations realized in $\mathcal{Q}_{n}$ and in $\mathcal{P}_{n}$ are actually the same. ${ }^{[*]}$

\section{B. Fock Quantization}

One can put forward a Schrödinger representation of the CCR's on a Hilbert space $\mathcal{H}=L^{2}(\mathcal{Q}, \mu)$ of square integrable (complex-valued) functions on the infinite dimensional linear space $\mathcal{Q}$ given by the direct sum of all the spaces $\mathcal{Q}_{n}$. For the sake of simplicity in the presentation, we will drop from now on the $n=0$ mode. ${ }^{[\dagger]}$ The measure $\mu$ is the Gaussian measure on $\mathcal{Q}$, obtained from the product of the 1-dimensional Gaussian measures, one per each degree of freedom, defined by the frequencies $\omega_{n}$. More precisely, the measure is

$$
\mathrm{d} \mu=\prod_{n, \ell, m}\left(\sqrt{\frac{\omega_{n}}{\pi}} e^{-\omega_{n} q_{n \ell m}^{2}} \mathrm{~d} q_{n \ell m}\right) .
$$

The ( $\mu$-compatible) basic operators of configuration and momentum act as multiplicative and derivative operators, respectively,

$$
\hat{q}_{n \ell m} \Psi=q_{n \ell m} \Psi, \quad \hat{p}_{n \ell m} \Psi=-i \frac{\partial}{\partial q_{n \ell m}} \Psi+i \omega_{n} q_{n \ell m} \Psi
$$

where $\Psi \in \mathcal{H}$ is arbitrary.

The constructed representation is just the one which is naturally associated with the free massless field. In this respect, let us point out that the above representation is defined

[*] This means that the matrices representing $S O(4)$-rotations are the same in $\mathcal{Q}_{n}$ and $\mathcal{P}_{n}$, if one adopts the respective bases provided by $\left\{q_{n \ell m}\right\}$ and $\left\{p_{n \ell m}\right\}$.

[ $\dagger$ ] It is a single mode, decoupled from the remaining degrees of freedom, and which can be quantized, at least for nonnegative mass functions $f(t)$, using the standard Schrödinger representation, with the Lebesgue measure on $\mathbb{R}$. Besides, the action of $S O(4)$ on this mode is trivial. Therefore, provided that $f(t)$ is nonnegative, the $n=0$ mode plays no role in the discussion of unitarity and uniqueness of the quantum representation. The full Hilbert space for the scalar field is then the tensor product of $L^{2}\left(\mathbb{R}, d q_{000}\right)$ with $\mathcal{H}$. 
by a complex structure $j_{0}$ on the canonical phase space which takes the form [3]

$$
j_{0}\left(\begin{array}{l}
\varphi \\
P
\end{array}\right)=\left(\begin{array}{cc}
0 & -(-h \Delta)^{-1 / 2} \\
(-h \Delta)^{1 / 2} & 0
\end{array}\right)\left(\begin{array}{l}
\varphi \\
P
\end{array}\right) .
$$

Let us remind that a complex structure is the mathematical structure that encodes the ambiguity which is physically relevant in the Fock quantization (or, equivalently, in its Schrödinger counterpart). A complex structure $J$ is a symplectic transformation in phase space that is compatible with the symplectic structure [in the sense that the combination $\Omega(J \cdot, \cdot)$ provides a positive definite bilinear map], and such that its square equals minus the identity, $J^{2}=-1$ (see Refs. [2, 3, 25] for details on the way in which a complex structure determines a Fock representation -and/or the corresponding Schrödinger one).

The complex structure (15) is obviously invariant under the action of $S O(4)$, a fact which immediately leads to a unitary implementation of the symmetry group. Of course, the complex structure is also time-translation invariant, a property which ensures a unitary implementation of the free field dynamics. Note however that no dynamically invariant complex structure is expected to exist when the field equation (21) is considered, for a generic function $f(t)$. We will then follow the strategy of considering $S O(4)$-invariant complex structures (there are plenty of those, as we will see) which, rather than being invariant under the evolution, allow one at least to obtain a unitary implementation of the field dynamics.

In order to discuss whether the dynamics is unitary, it is more convenient to replace the canonical variables $\left(q_{n \ell m}, p_{n \ell m}\right)$ with the annihilation-like variables

$$
a_{n \ell m}=\frac{1}{\sqrt{2 \omega_{n}}}\left(\omega_{n} q_{n \ell m}+i p_{n \ell m}\right), \quad n=1,2, \ldots
$$

and the creation-like variables provided by their complex conjugates $a_{n \ell m}^{*}$. We will use this set of complex variables as coordinates for the inhomogeneous sector $(n \neq 0)$ of the canonical phase space. Note that the variables (16) correspond to the annihilation operators of the considered quantum representation. Precisely because of that, the complex structure $j_{0}$ given in Eq. (15) takes a particularly simple form with respect to these 
variables: it is block diagonal and completely defined by

$$
j_{0}\left(a_{n \ell m}\right)=i a_{n \ell m}, \quad j_{0}\left(a_{n \ell m}^{*}\right)=-i a_{n \ell m}^{*}, \quad \forall n, \ell, m
$$

As a consequence of the decoupling between degrees of freedom, the finite transformations generated by the dynamics are linear symplectic transformations which can be decomposed in $2 \times 2$ blocks, one for each fixed pair $\left(a_{n \ell m}, a_{n \ell m}^{*}\right)$. Thus, the classical evolution of the annihilation and creation-like variables from time $t_{0}$ to time $t$ is totally determined by a discrete set of $2 \times 2$ matrices $\mathcal{U}_{n \ell m}\left(t, t_{0}\right)$. Furthermore, since the dynamical equations (10) are independent of $\ell$ and $m$, the same is true for the evolution matrices, i.e.,

$$
\begin{gathered}
\left(\begin{array}{c}
a_{n \ell m}(t) \\
a_{n \ell m}^{*}(t)
\end{array}\right)=\mathcal{U}_{n \ell m}\left(t, t_{0}\right)\left(\begin{array}{c}
a_{n \ell m}\left(t_{0}\right) \\
a_{n \ell m}^{*}\left(t_{0}\right)
\end{array}\right)=\mathcal{U}_{n}\left(t, t_{0}\right)\left(\begin{array}{c}
a_{n \ell m}\left(t_{0}\right) \\
a_{n \ell m}^{*}\left(t_{0}\right)
\end{array}\right), \\
\mathcal{U}_{n \ell m}\left(t, t_{0}\right):=\left(\begin{array}{cc}
\alpha_{n \ell m}\left(t, t_{0}\right) & \beta_{n \ell m}\left(t, t_{0}\right) \\
\beta_{n \ell m}^{*}\left(t, t_{0}\right) & \alpha_{n \ell m}^{*}\left(t, t_{0}\right)
\end{array}\right)=\mathcal{U}_{n}\left(t, t_{0}\right):=\left(\begin{array}{cc}
\alpha_{n}\left(t, t_{0}\right) & \beta_{n}\left(t, t_{0}\right) \\
\beta_{n}^{*}\left(t, t_{0}\right) & \alpha_{n}^{*}\left(t, t_{0}\right)
\end{array}\right) .
\end{gathered}
$$

Here, $\alpha_{n}\left(t, t_{0}\right)$ and $\beta_{n}\left(t, t_{0}\right)$ are Bogoliubov coefficients for the evolution equations (10), which therefore depend on the specific function $f(t)$. Since $\mathcal{U}_{n}\left(t, t_{0}\right)$ provides a symplectomorphism, one can easily check that, for any value of $t_{0}$ and $\forall n,\left|\alpha_{n}\left(t, t_{0}\right)\right|^{2}-\left|\beta_{n}\left(t, t_{0}\right)\right|^{2}=1$ for all $t \in \mathbb{I}$.

\section{UNITARY DYNAMICS}

We will now show that the field dynamics is implemented as a unitary transformation in the quantum representation defined by $j_{0}$ even if the field possesses a nonconstant mass, at least under quite mild conditions on the time dependence of this mass and in spite of the loss of time invariance of the system. 


\section{A. Conditions for a unitary implementation}

Let us consider the operators $\hat{a}_{n \ell m}$, related to the operators $\hat{q}_{n \ell m}$ and $\hat{p}_{n \ell m}$ [introduced in Eq. (14)] by means of the linear relations (16). As mentioned above, these are the annihilation operators of the $j_{0}$-representation.

In the Heisenberg picture, time evolution in the quantum theory is, in principle, given again by the Bogoliubov transformation (19), what means that the operators $\left(\hat{a}_{n \ell m}, \hat{a}_{n \ell m}^{\dagger}\right)$ at time $t_{0}$ evolve to operators $\left(\hat{a}_{n \ell m}(t), \hat{a}_{n \ell m}^{\dagger}(t)\right)$ at time $t$ according to the same transformation:

$$
\left(\begin{array}{l}
\hat{a}_{n \ell m}(t) \\
\hat{a}_{n \ell m}^{\dagger}(t)
\end{array}\right)=\left(\begin{array}{ll}
\alpha_{n}\left(t, t_{0}\right) & \beta_{n}\left(t, t_{0}\right) \\
\beta_{n}^{*}\left(t, t_{0}\right) & \alpha_{n}^{*}\left(t, t_{0}\right)
\end{array}\right)\left(\begin{array}{l}
\hat{a}_{n \ell m} \\
\hat{a}_{n \ell m}^{\dagger}
\end{array}\right)
$$

The question that we want to address is whether or not these transformations as a whole $(\forall n, \ell$, and $m$ ) can be unitarily implemented, i.e., if they correspond to a unitary transformation $U\left(t, t_{0}\right)$ in the Hilbert space $\mathcal{H}$.

The main advantage of using the variables (16) is that the condition for unitary implementability can be rephrased in a simple form. In general, a symplectic transformation $R$ can be unitarily implemented on a Fock representation, constructed from a complex structure $J$, if and only if $R+J R J$ is an operator of the Hilbert-Schmidt type on the corresponding 1-particle Hilbert space [26, 27]. Equivalently, $R$ is implementable as a unitary transformation if and only if $J-R J R^{-1}$ is a Hilbert-Schmidt operator (note that each of these conditions ensures that the representations defined by the complex structures $J$ and $R J R^{-1}$ are unitarily equivalent, something which is essentially the definition of unitary implementability). In the case of the family of symplectic transformations defined by the classical dynamics, specified by the matrices $\mathcal{U}_{n}\left(t, t_{0}\right)$, the Hilbert-Schmidt condition for a unitary implementation in the $j_{0}$-Fock representation becomes a square summability condition on the coefficients $\beta_{n \ell m}$, namely,

$$
\sum_{n=1}^{\infty} \sum_{\ell=0}^{n} \sum_{m=-\ell}^{\ell}\left|\beta_{n \ell m}\left(t, t_{0}\right)\right|^{2}<\infty \quad \forall t \in \mathbb{I},
$$

given a fixed reference time $t_{0}$. 
In the present case, owing to the independence of the equations of motion (10) with respect to $\ell$ and $m$, we have

$$
\sum_{n=1}^{\infty} \sum_{\ell=0}^{n} \sum_{m=-\ell}^{\ell}\left|\beta_{n \ell m}\left(t, t_{0}\right)\right|^{2}=\sum_{n=1}^{\infty} g_{n}\left|\beta_{n}\left(t, t_{0}\right)\right|^{2}
$$

where the degeneracy factor $g_{n}=(n+1)^{2}$ counts the number of degrees of freedom with the same dynamics. Thus, the unitary implementability condition becomes a square summability condition for the sequences $\sqrt{g_{n}} \beta_{n}\left(t, t_{0}\right)$, i.e.,

$$
\sum_{n=1}^{\infty} g_{n}\left|\beta_{n}\left(t, t_{0}\right)\right|^{2}<\infty
$$

where the coefficients $\beta_{n}$ are those corresponding to the differential equations

$$
\ddot{q}_{n}+\left(\omega_{n}^{2}+f\right) q_{n}=0
$$

\section{B. Asymptotic dynamics and unitarity}

In order to elucidate whether condition (23) is fulfilled, we are interested in investigating the large $n$-limit of the coefficients $\beta_{n}$, and therefore the behavior of the solutions to the differential equation (24) for large $n$.

Let us start by writing the general solution to that equation of motion in the form

$$
q_{n}(t)=A_{n} \exp \left[\omega_{n} \Theta_{n}(t)\right]+A_{n}^{*} \exp \left[\omega_{n} \Theta_{n}^{*}(t)\right]
$$

For each $n, A_{n}$ is a complex constant related to the initial conditions and $\Theta_{n}$ is a particular (complex) solution of the equation

$$
\omega_{n} \ddot{\Theta}_{n}+\omega_{n}^{2} \dot{\Theta}_{n}^{2}+\omega_{n}^{2}+f=0
$$

which is obtained from Eqs. (24) and (25).

We fix the arbitrariness in the solution $\Theta_{n}$ of the above equation by means of the initial conditions $\Theta_{n}\left(t_{0}\right)=0$ and $\dot{\Theta}_{n}\left(t_{0}\right)=-i, \forall n$. A simple analysis shows that this is always possible (see Ref. [7] for details). This choice is motivated by the free massless scalar field 
case [i.e., the case with $f(t)=0$ ], for which $\dot{\Theta}_{n}=-i$ is satisfied not only initially, but at all times.

By working out the relation between the canonical data $\left(q_{n}\left(t_{0}\right), \dot{q}_{n}\left(t_{0}\right)\right)$ and the complex constant $A_{n}$ in Eq. (25), one can easily obtain the evolution matrices in terms of the canonical variables. Finally, changing from those variables to the annihilation and creation-like variables, it is straightforward to deduce the expression of the Bogoliubov coefficients $\alpha_{n}\left(t, t_{0}\right)$ and $\beta_{n}\left(t, t_{0}\right)$ appearing in the evolution matrices (19)). We get

$$
\begin{aligned}
\alpha_{n}\left(t, t_{0}\right) & =\frac{1}{2} e^{\omega_{n} \Theta_{n}(t)}\left[1+i \dot{\Theta}_{n}(t)\right], \\
\beta_{n}\left(t, t_{0}\right) & =\frac{1}{2} e^{\omega_{n} \Theta_{n}^{*}(t)}\left[1+i \dot{\Theta}_{n}^{*}(t)\right] .
\end{aligned}
$$

As an aside we note that, in the equations of motion (24), the term $\omega_{n}^{2}$ dominates over the $n$-independent mass term $f(t)$ in the limit of large $n$; we thus expect that the solutions $q_{n}(t)$ converge to those corresponding to the free massless case (for the same initial conditions), at least for sufficiently regular functions $f(t)$.

In order to see that the asymptotic corrections to this behavior for large $n$ do not affect unitarity, we only need to analyze the unitary implementability condition (23), which translates now into

$$
\sum_{n=1}^{\infty} g_{n} e^{2 \omega_{n} \Re\left[\Theta_{n}^{*}(t)\right]}\left|1+i \dot{\Theta}_{n}^{*}(t)\right|^{2}<\infty \quad \forall t \in \mathbb{I} .
$$

In the following we will therefore focus on the behavior of $\Theta_{n}$ for large $n$.

It is convenient to write the functions $\dot{\Theta}_{n}$ in the form

$$
\dot{\Theta}_{n}=-i+\frac{W_{n}}{\omega_{n}}
$$

so that the expected asymptotic limit, for large $n$, is already singled out. From Eq. (26), it follows that the functions $W_{n}$ satisfy the first-order differential equations

$$
\dot{W}_{n}=2 i \omega_{n} W_{n}-W_{n}^{2}-f
$$

with the initial conditions $W_{n}\left(t_{0}\right)=0$, deduced from the above conditions on the functions $\dot{\Theta}_{n}$. In addition, recalling that $\Theta_{n}\left(t_{0}\right)=0$, we obtain

$$
\Theta_{n}=-i\left(t-t_{0}\right)+\int_{t_{0}}^{t} \mathrm{~d} \bar{t} \frac{W_{n}(\bar{t})}{\omega_{n}} .
$$


Condition (29) can then be rewritten as

$$
\sum_{n=1}^{\infty} g_{n} e^{2 \int_{t_{0}}^{t} \Re\left[W_{n}\right]} \frac{\left|W_{n}(t)\right|^{2}}{\omega_{n}^{2}}<\infty \quad \forall t \in \mathbb{I} .
$$

We will now prove that, in the large $n$-limit, the desired solutions to Eq. (31) possess "ultraviolet modes" of order $1 / n$, such that the unitarity condition (33) is satisfied. The argument goes as follows. In the limit of large $n$, the term $W_{n}^{2}$ in Eq. (31) is dominated by the term linear in $W_{n}$, whose coefficient grows with $n$ [see Eq. (11)]. One can then start by neglecting the quadratic term $W_{n}^{2}$, show that the resulting linear equation admits solutions $\bar{W}_{n}$ of order $1 / n$, and check that, in the limit of large $n$, the contribution of the quadratic term for such solutions is in fact negligible in the original differential equation.

Let us then consider the linear differential equation obtained from Eq. (31) after removing the quadratic term:

$$
\dot{\bar{W}}_{n}=2 i \omega_{n} \bar{W}_{n}-f
$$

The solution to Eq. (34) satisfying the initial condition $W_{n}\left(t_{0}\right)=0$ is

$$
\bar{W}_{n}(t)=-\exp \left(2 i \omega_{n} t\right) \int_{t_{0}}^{t} \mathrm{~d} \bar{t} f(\bar{t}) \exp \left(-2 i \omega_{n} \bar{t}\right)
$$

and an integration by parts leads to

$$
\bar{W}_{n}(t)=-\frac{i f(t)}{2 \omega_{n}}+\frac{i f\left(t_{0}\right) e^{2 i \omega_{n}\left(t-t_{0}\right)}}{2 \omega_{n}}-\frac{\exp \left(2 i \omega_{n} t\right)}{2 i \omega_{n}} \int_{t_{0}}^{t} \mathrm{~d} \bar{t} \dot{f}(\bar{t}) \exp \left(-2 i \omega_{n} \bar{t}\right) .
$$

The absolute value of the last term in Eq. (36) is bounded by $\int_{t_{0}}^{t} \mathrm{~d} t|\dot{f}| /\left(2 \omega_{n}\right)$. One therefore concludes that there is a function $C(t)$, independent of $n$, such that the absolute value of the solutions (35) is bounded by $C(t) / \omega_{n}$. To reach this conclusion, it is sufficient that the derivative of the function $f(t)$ exists and is integrable in every closed interval $\left[t_{0}, t\right]\left(\right.$ or $\left.\left[t, t_{0}\right]\right)$ of $\mathbb{I}$.

We now return to the original differential equation (31). Since the quadratic term $\bar{W}_{n}^{2}$ is bounded in absolute value by $C(t)^{2} / \omega_{n}^{2}$, it is negligible, in particular compared with the linear term in Eq. (31). Therefore, the functions $\bar{W}_{n}(t)$ defined in formula (35) can be taken as asymptotic solutions of Eq. (31) in the limit of large $n$, up to higher order corrections. 
Finally, we can show that the unitary implementability condition is indeed fulfilled. From our arguments above and the fact that $g_{n}=(n+1)^{2}$ and $\omega_{n}^{2}=n(n+2)$, it is clear that the summand in condition (33) has the same asymptotic behavior as $\left|W_{n}\right|^{2}$ for large $n$. But $\left|W_{n}\right|^{2}$ is certainly summable since, apart from subdominant terms which do not affect the summability, $\left|W_{n}\right|^{2}$ is bounded by $C(t)^{2} / \omega_{n}^{2}$. So, the unitary implementability condition is satisfied.

\section{UNIQUENESS OF THE QUANTIZATION}

We have just seen that there is a Fock representation, determined by an $S O(4)$-invariant complex structure, which allows a unitary dynamics for the scalar field with a time dependent mass. The question we want to address now is that of uniqueness. Are there distinct representations with the same properties? We will show that the answer is in the negative. Although there are in fact infinitely many distinct, i.e., non-unitarily equivalent, $S O(4)$-invariant Fock representations, we will show that the requirement of a unitary implementation of the field dynamics selects in fact a unique unitary equivalence class of representations.

\section{A. Invariant complex structures}

Let us restrict our considerations from now on exclusively to complex structures $J$ that are invariant under the action of the symmetry group, i.e., such that $T^{-1} J T=J$, $\forall T \in S O(4)$. We will refer to such complex structures simply as invariant ones.

A characterization of invariant complex structures is easily obtained by employing Schur's lemma as follows (see Ref. [11]). The phase space $\Gamma$ can be decomposed as the direct sum

$$
\Gamma=\bigoplus_{n} \Gamma_{n}
$$

with $\Gamma_{n}:=\mathcal{Q}_{n} \oplus \mathcal{P}_{n}$. Then, a simple application of Schur's lemma [28] leads to the conclusion that an invariant complex structure $J$ is block diagonal with respect to the 
above decomposition, i.e., an invariant complex structure $J: \Gamma \rightarrow \Gamma$ is of the form

$$
J=\bigoplus_{n} J_{n}
$$

where $J_{n}: \Gamma_{n} \rightarrow \Gamma_{n}$ are arbitrary $S O(4)$-invariant complex structures. Consider now a basis in each space $\Gamma_{n}$, for instance the basis provided by the variables $q_{n \ell m}$ and the corresponding momenta $p_{n \ell m}$. Then, each $J_{n}$ corresponds to a matrix, characterized by four square blocks: $J_{n}^{q q}, J_{n}^{q p}, J_{n}^{p q}$, and $J_{n}^{p p}$, which connect respectively $\mathcal{Q}_{n}$ to itself, $\mathcal{Q}_{n}$ to $\mathcal{P}_{n}, \mathcal{P}_{n}$ to $\mathcal{Q}_{n}$, and $\mathcal{P}_{n}$ to itself. The invariance conditions on those blocks reads

$$
\begin{array}{ll}
D_{q}(T) J_{n}^{q q}=J_{n}^{q q} D_{q}(T), & D_{p}(T) J_{n}^{q p}=J_{n}^{q p} D_{q}(T), \\
D_{q}(T) J_{n}^{p q}=J_{n}^{p q} D_{p}(T), & D_{p}(T) J_{n}^{p p}=J_{n}^{p p} D_{p}(T),
\end{array}
$$

where $T$ is an arbitrary $S O(4)$ transformation and $D_{q}$ (respectively $D_{p}$ ) denotes the matrix representation realized in $\mathcal{Q}_{n}\left(\mathcal{P}_{n}\right)$. Actually, according to our comments at the end of Sec. 2A, the matrices $D_{q}(T)$ and $D_{p}(T)$ must coincide for each $T$, so that conditions (39, 40) become invariance conditions for matrices in the considered $(n+1)^{2}$-dimensional representation of $S O(4)$, which is known to be irreducible. Then, again by Schur's lemma, each of the matrices $J_{n}^{q q}, J_{n}^{q p}, J_{n}^{p q}$, and $J_{n}^{p p}$ must be proportional to the identity matrix $\mathbf{I}$, namely $J_{n}^{q q}=a_{n} \mathbf{I}, J_{n}^{p q}=b_{n} \mathbf{I}, J_{n}^{q p}=c_{n} \mathbf{I}$, and $J_{n}^{p p}=d_{n} \mathbf{I}$, with $a_{n}, b_{n}, c_{n}$, and $d_{n}$ certain complex numbers.

In fact, the above conclusion applies in principle to complex representations only, whereas we are dealing instead with the real spaces $\mathcal{Q}_{n}$ and $\mathcal{P}_{n}$. Nonetheless, it is clear that every invariant complex structure in these real spaces can be obtained from the restriction (to such real subspaces) of an invariant complex structure defined on their corresponding complex counterparts. The above result is therefore valid in our case as well. Moreover, since only real matrices have a well defined action on real subspaces, the proportionality coefficients $a_{n}, b_{n}, c_{n}$, and $d_{n}$ must be all real. Finally, since $J$ is a complex structure, one can conclude that, for all $n$, these coefficients, organized in the 
matrix

$$
\left(\begin{array}{ll}
a_{n} & b_{n} \\
c_{n} & d_{n}
\end{array}\right)
$$

must form a complex structure in two dimensions.

Once we have characterized the invariant complex structures it is convenient, for what follows, to relate them to the complex structure $j_{0}$ which determines the representation (13, 14) of Sec. 2B. We start by decomposing $\Gamma_{n}$ in terms of independent degrees of freedom, i.e., as the direct sum

$$
\Gamma_{n}=\bigoplus_{\ell m} \Gamma_{n \ell m}
$$

where each $\Gamma_{n \ell m}$ is the 2-dimensional symplectic space spanned by $q_{n \ell m}$ and $p_{n \ell m}$. It is clear from the above description of the invariant complex structures that each $J_{n}$ in Eq. (38) can be further decomposed in the block diagonal form

$$
J_{n}=\bigoplus_{\ell m} J_{n \ell m}
$$

and that each transformation $J_{n \ell m}$, for fixed $n$, is given by the same matrix, namely the matrix (41), independent of $\ell$ and $m$.

In each space $\Gamma_{n \ell m}$, we now change from the real basis $\left(q_{n \ell m}, p_{n \ell m}\right)$ to the complex variables $a_{n \ell m}(16)$ and $a_{n \ell m}^{*}$, and call $j_{n}$ the matrix corresponding to the $2 \times 2$ complex structure (41) in the new basis. It is not difficult to show [5] that each $j_{n}$ is related to $j_{0_{n}}$ via a symplectic transformation $\mathcal{K}_{n}: j_{n}=\mathcal{K}_{n} j_{0_{n}} \mathcal{K}_{n}^{-1}$, where $j_{0_{n}}$ is the basic $2 \times 2$ block for the complex structure $j_{0}$, which in the variables $\left(a_{n \ell m}, a_{n \ell m}^{*}\right)$ takes the form

$$
j_{0 n}=\left(\begin{array}{cc}
i & 0 \\
0 & -i
\end{array}\right)
$$

In addition, we adopt the following notation for the $2 \times 2$ symplectic transformation $\mathcal{K}_{n}$ :

$$
\mathcal{K}_{n}=\left(\begin{array}{cc}
\kappa_{n} & \lambda_{n} \\
\lambda_{n}^{*} & \kappa_{n}^{*}
\end{array}\right),
$$

with $\left|\kappa_{n}\right|^{2}-\left|\lambda_{n}\right|^{2}=1, \forall n \in \mathbb{N}^{+}$. 
In other words, any invariant complex structure $J$ is related to $j_{0}$ by a correspondence of the form $J=K j_{0} K^{-1}$, where $K$ is a symplectic transformation which is block diagonal with respect to the decomposition (42), and whose basic blocks (independent of $\ell$ and $m$ ) are the matrices $\mathcal{K}_{n}$ (45). In particular, the information determining a given invariant complex structure $J$ is encoded in the corresponding sequence of matrices $\mathcal{K}_{n}$.

\section{B. Unitary dynamics and equivalence of representations}

A given symplectic transformation $R$ admits a unitary implementation with respect to the complex structure $J=K j_{0} K^{-1}$ if and only if the transformation $K^{-1} R K$ is unitarily implementable with respect to $j_{0}$ [5]. Hence, the time evolution, specified by the sequence of matrices $\left\{\mathcal{U}_{n}\right\}$ (19), is unitarily implementable with respect to the Fock representation determined by an invariant complex structure $J$ if and only if the $j_{0^{-}}$ representation admits a unitary implementation of the symplectomorphism specified by the sequence $\left\{\mathcal{K}_{n}^{-1} \mathcal{U}_{n} \mathcal{K}_{n}\right\}$, where $\left\{\mathcal{K}_{n}\right\}$ corresponds to $J$.

It is worth noticing that, like the original evolution matrices $\mathcal{U}_{n}$, the transformed ones $\mathcal{K}_{n}^{-1} \mathcal{U}_{n} \mathcal{K}_{n}$ are independent of the additional indices $\ell$ and $m$, a property which is due precisely to the similar independence displayed by the matrices of the symplectic transformation $K$ that characterizes an invariant complex structure. Thus, the transformed evolution matrices have again the general form (19), and a straightforward computation allow us to relate the new matrix elements with $\alpha_{n}$ and $\beta_{n}$, given in Eqs. (27, 28). In particular, for the new beta coefficients, which we will call $\beta_{n}^{J}$, we obtain:

$$
\beta_{n}^{J}\left(t, t_{0}\right)=\left(\kappa_{n}^{*}\right)^{2} \beta_{n}\left(t, t_{0}\right)-\lambda_{n}^{2} \beta_{n}^{*}\left(t, t_{0}\right)+2 i \kappa_{n}^{*} \lambda_{n} \Im\left[\alpha_{n}\left(t, t_{0}\right)\right], \quad \forall t \in \mathbb{I} .
$$

As expected, the unitary implementability condition for the field dynamics, with respect to the complex structure $J=K j_{0} K^{-1}$, amounts to the square summability of the sequences $\sqrt{g_{n}} \beta_{n}^{J}\left(t, t_{0}\right)$, summability which replaces the corresponding condition (23).

On the other hand, we recall that the Fock representation specified by $J=K j_{0} K^{-1}$ and the $j_{0}$-representation are unitarily equivalent if and only if the transformation $K$ can 
be unitarily implemented, a requirement which reduces to the condition

$$
\sum_{n \ell m}\left|\lambda_{n}\right|^{2}=\sum_{n=1}^{\infty} g_{n}\left|\lambda_{n}\right|^{2}<\infty
$$

where the coefficients $\lambda_{n}$ are the ones appearing in the matrices $\mathcal{K}_{n}$ given in Eq. (45) (see Ref. [5] for more details). In the remaining of the present section, we will show that, if the

sequences $\left\{\sqrt{g_{n}} \beta_{n}^{J}\left(t, t_{0}\right)\right\}$ are square summable $\forall t \in \mathbb{I}$, then the same must happen with the sequence $\left\{\sqrt{g_{n}} \lambda_{n}\right\}$. So, we will prove that any other representation, defined by an invariant complex structure and allowing a unitary implementation of the field dynamics, is in fact unitarily equivalent to the $j_{0}$-Fock representation.

\section{Proof of uniqueness}

According to our discussion, we restrict our considerations to any of the invariant complex structures $J$ such that $\left\{\sqrt{g_{n}} \beta_{n}^{J}\left(t, t_{0}\right)\right\}$ is square summable $\forall t \in \mathbb{I}$. Since $\left|\kappa_{n}\right|>1$, the sequences

$$
\frac{\sqrt{g_{n}} \beta_{n}^{J}\left(t, t_{0}\right)}{\left(\kappa_{n}^{*}\right)^{2}}=\sqrt{g_{n}} \beta_{n}\left(t, t_{0}\right)-\left(\frac{\lambda_{n}}{\kappa_{n}^{*}}\right)^{2} \sqrt{g_{n}} \beta_{n}^{*}\left(t, t_{0}\right)+2 i \sqrt{g_{n}}\left(\frac{\lambda_{n}}{\kappa_{n}^{*}}\right) \Im\left[\alpha_{n}\left(t, t_{0}\right)\right]
$$

are then also square summable. Moreover, from the fact that $\left|\kappa_{n}\right|^{2}-\left|\lambda_{n}\right|^{2}=1$ it follows that the sequence $\left\{\lambda_{n} / \kappa_{n}^{*}\right\}$ is bounded, and since we already know that the sequence $\left\{\sqrt{g_{n}} \beta_{n}\left(t, t_{0}\right)\right\}$ is square summable, we can guarantee that

$$
\left\{\sqrt{g_{n}} \beta_{n}\left(t, t_{0}\right)-\left(\frac{\lambda_{n}}{\kappa_{n}^{*}}\right)^{2} \sqrt{g_{n}} \beta_{n}^{*}\left(t, t_{0}\right)\right\}
$$

is a square summable sequence $\forall t \in \mathbb{I}$. Given that the set of square summable sequences is a linear space, one is led to the conclusion that the sequence

$$
\left\{\sqrt{g_{n}} \frac{\lambda_{n}}{\kappa_{n}^{*}} \Im\left[\alpha_{n}\left(t, t_{0}\right)\right]\right\}
$$

is square summable $\forall t \in \mathbb{I}$ as well.

In order to proceed further, we write $\Im\left[\alpha_{n}\right]$ using Eqs. (27) and (130) in the form

$$
\Im\left[\alpha_{n}\right]=e^{\omega_{n} \Re\left[\Theta_{n}\right]}\left(1-\frac{\Im\left[W_{n}\right]}{2 \omega_{n}}\right) \sin \left(\omega_{n} \Im\left[\Theta_{n}\right]\right)+e^{\omega_{n} \Re\left[\Theta_{n}\right]} \frac{\Re\left[W_{n}\right]}{2 \omega_{n}} \cos \left(\omega_{n} \Im\left[\Theta_{n}\right]\right) .
$$


On the other hand, our asymptotic analysis about the square summability of $\beta_{n}$, carried out in Sec. 3B, ensures that both

$$
\sqrt{g_{n}} e^{\omega_{n} \Re\left[\Theta_{n}\right]} \frac{\Im\left[W_{n}\right]}{\omega_{n}} \text { and } \sqrt{g_{n}} e^{\omega_{n} \Re\left[\Theta_{n}\right]} \frac{\Re\left[W_{n}\right]}{\omega_{n}}
$$

provide square summable sequences. Returning then to the sequence (50), one concludes that the contribution from the terms proportional to the factors in Eq. (52) gives a square summable part, since the remaining factors, namely $\lambda_{n} / \kappa_{n}^{*}$ and the trigonometric functions, are bounded. Therefore, invoking again the linearity of the space of square summable sequences, and using in addition that the sequence $\left\{e^{\omega_{n} \Re\left[\Theta_{n}\right]}\right\}$ is bounded from below because $\omega_{n} \Re\left[\Theta_{n}\right]$ is at least of order $1 / n$ according to our asymptotic analysis, we conclude that, for any invariant complex structure which leads to a unitary dynamics, the sequence

$$
\left\{\sqrt{g_{n}} \frac{\lambda_{n}}{\kappa_{n}^{*}} \sin \left(\omega_{n} \Im\left[\Theta_{n}\right]\right)\right\}
$$

has to be square summable $\forall t \in \mathbb{I}$.

We next analyze the trigonometric function appearing in this last expression. Recalling again our asymptotic analysis of Sec. $3 \mathrm{~B}$, and provided that the second derivative of the function $f(t)$ exists and is integrable in every closed subinterval of $\mathbb{I}$, we can integrate by parts the last term in Eq. (36) to show that, apart from terms of order $1 / n^{2}, \Im\left[W_{n}\right]$ is given by

$$
-\frac{f(t)}{2 \omega_{n}}+\frac{f\left(t_{0}\right)}{2 \omega_{n}} \cos \left[2 \omega_{n}\left(t-t_{0}\right)\right]
$$

Since

$$
\int_{t_{0}}^{t} \mathrm{~d} \bar{t} \cos \left[2 \omega_{n}\left(\bar{t}-t_{0}\right)\right]=\frac{\sin \left[2 \omega_{n}\left(t-t_{0}\right)\right]}{2 \omega_{n}}
$$

one can check then from Eq. (32) that, except for a remaining contribution of order $1 / n^{2}$, $\Im\left[\omega_{n} \Theta_{n}\left(t, t_{0}\right)\right]$ behaves like

$$
\omega_{n}\left(t_{0}-t\right)+\int_{t}^{t_{0}} \mathrm{~d} \bar{t} \frac{f(\bar{t})}{2 \omega_{n}}
$$

Hence, in the large $n$-limit we obtain

$$
\sin \left(\omega_{n} \Im\left[\Theta_{n}\right]\right)=-\sin \left[\omega_{n}\left(t-t_{0}\right)+\int_{t_{0}}^{t} \mathrm{~d} \bar{t} \frac{f(\bar{t})}{2 \omega_{n}}\right]+o\left(\frac{1}{n^{2}}\right),
$$


where $o\left(1 / n^{2}\right)$ denotes a contribution of asymptotic order $1 / n^{2}$. After multiplication by $\sqrt{g_{n}}\left(\lambda_{n} / \kappa_{n}^{*}\right)$, the last term gives a contribution of order $1 / n$ which is square summable. Therefore, it follows that the sequence

$$
\left\{\sqrt{g_{n}} \frac{\lambda_{n}}{\kappa_{n}^{*}} \sin \left[\omega_{n}\left(t-t_{0}\right)+\int_{t_{0}}^{t} \mathrm{~d} \bar{t} \frac{f(\bar{t})}{2 \omega_{n}}\right]\right\}
$$

is square summable $\forall t \in \mathbb{I}$.

It is convenient to introduce now the shifted time $T:=t-t_{0}$, and rewrite the elements of the sequence (58) in the form

$$
\sqrt{g_{n}} \frac{\lambda_{n}}{\kappa_{n}^{*}} \sin \left[\omega_{n} T+\int_{0}^{T} \mathrm{~d} \bar{t} \frac{f\left(\bar{t}+t_{0}\right)}{2 \omega_{n}}\right]
$$

Then, the function

$$
z(T):=\lim _{M \rightarrow \infty} \sum_{n=1}^{M} g_{n} \frac{\left|\lambda_{n}\right|^{2}}{\left|\kappa_{n}\right|^{2}} \sin ^{2}\left[\omega_{n} T+\int_{0}^{T} \mathrm{~d} \bar{t} \frac{f\left(\bar{t}+t_{0}\right)}{2 \omega_{n}}\right]
$$

exists for all $T$ in the domain $\overline{\mathbb{I}}$, obtained from the domain $\mathbb{I}$ of $t$ after the shift by $t_{0}$. In particular, $z(T)$ is well defined on some closed subinterval of the form $\overline{\mathbb{I}}_{L}=[a, a+L] \subseteq \overline{\mathbb{I}}$ (for a suitable choice of the time $a$ ), where $L$ is some finite positive number strictly smaller than the length of $\mathbb{I}$.

Let us now apply Luzin's theorem [29] to the function $z(T)$. This theorem guarantees that, for every $\delta>0$, there exist: i) a measurable set $E_{\delta} \subset \overline{\mathbb{I}}_{L}$ such that its complement $\bar{E}_{\delta}$ with respect to $\overline{\mathbb{I}}_{L}$ satisfies $\int_{\bar{E}_{\delta}} \mathrm{d} T<\delta$, and ii) a function $F_{\delta}(T)$ which is continuous on $\overline{\mathbb{I}}_{L}$ and coincides with $z(T)$ in $E_{\delta}$. In particular, defining $I_{\delta}:=\int_{E_{\delta}} F_{\delta}(T) \mathrm{d} T$, which is a finite real number, we obtain from Luzin's theorem that

$$
\sum_{n=1}^{M} g_{n} \frac{\left|\lambda_{n}\right|^{2}}{\left|\kappa_{n}\right|^{2}} \int_{E_{\delta}} \sin ^{2}\left[\omega_{n} T+\int_{0}^{T} \mathrm{~d} \bar{t} \frac{f\left(\bar{t}+t_{0}\right)}{2 \omega_{n}}\right] \mathrm{d} T \leq \int_{E_{\delta}} z(T) \mathrm{d} T=I_{\delta}, \quad \forall M \in \mathbb{N}^{+} .
$$

In the appendix we prove that, actually, the above inequality allows one to demonstrate that the sequence $\left\{\sqrt{g_{n}} \lambda_{n} / \kappa_{n}\right\}$ is square summable. From this summability and the relation $\left|\kappa_{n}\right|^{2}-\left|\lambda_{n}\right|^{2}=1$, one can also see that the sequence $\left\{\kappa_{n}\right\}$ tends to 1 , and thus it is bounded. Therefore, the sequence $\left\{\sqrt{g_{n}} \lambda_{n}\right\}$ must be square summable, so that the 
condition of unitary equivalence between complex structures [see Eq. (477)] is indeed satisfied. This concludes the proof of uniqueness of the equivalence class of invariant complex structures which provide a unitary implementation of the dynamics.

\section{CONCLUSIONS AND EXTENSION TO OTHER MANIFOLDS: THE CASE OF $S^{2}$}

We have considered a free scalar field with a time dependent mass propagating in a $(1+3)$-spacetime with the topology of $\mathbb{I} \times S^{3}$, where $\mathbb{I}$ is an interval of the real line, provided with a product metric given by the standard metric on the three-sphere and the trivial metric on the time interval $\mathbb{I}$. Among the infinitely many possible Fock representations of the CCR's for this field, we have succeded to show that the representation which is naturally associated with the massless free case does not only incorporate the symmetries of the field equation, but implements the dynamics corresponding to the time varying mass as unitary transformations as well. More importantly, we have proved that, among all the Fock representations based on complex structures that are invariant under the group of symmetries of the field equation, the unitary equivalence class of the representation for the free massless case is in fact the unique class which supports a unitary dynamics. In this sense, the Fock quantization is unique once one demands a natural implementation of the symmetries of the field equation and that the dynamics be unitary.

The discussion that we have presented can be extended or generalized to cases in which the spatial manifold is not $S^{3}$ but another compact manifold. Let us assume that the negative of the Laplace-Beltrami operator for that manifold (a nonnegative operator) has also a discrete spectrum, with eigenvalues $\omega_{n}^{2}$ labeled in increasing order by the index $n=0,1, \ldots$, and such that $\omega_{n}$ tends to infinity when $n$ does so. Again, we call $g_{n}$ the dimension of each of the corresponding eigenspaces. It is not difficult to realize that the asymptotic analysis for large $n$ carried out in Sec. $3 \mathrm{~B}$ can be alternatively understood as an asymptotic analysis for large $\omega_{n}$, with asymptotic expansions in powers of $1 / \omega_{n}$. Then, from the arguments presented above (and recalling in particular that $W_{n}$ is of order $1 / \omega_{n}$ 
up to subdominant terms), one concludes that the complex structure of the free massless case leads to a Fock representation with a unitary implementation of the dynamics also in the presence of a time dependent mass (at least for a sufficiently regular time dependence) if and only if the sequence with elements $g_{n} / \omega_{n}^{4}$ is summable. On the other hand, as we have commented, the Fock representation associated with the free massless case clearly provides a natural unitary implementation of the symmetries of the Laplace-Beltrami operator.

Summarizing, the availability of the Fock representation of the free massless case in order to obtain an invariant vacuum and a quantum unitary dynamics when the mass varies in time is maintained for more general compact manifolds than $S^{3}$, and the relevant condition is the summability of the sequence formed by $g_{n} / \omega_{n}^{4}$. Concerning the uniqueness of this representation, modulo unitary equivalence, we note that the only additional property employed in our discussion of Sec. 4A and which has proved to be sufficient to guarantee uniqueness, is the irreducibility of the representation of the group of symmetries of the field equation in each of the eigenspaces of the Laplace-Beltrami operator. It is worth emphasizing that this condition suffices to reach uniqueness once the Fock representation which corresponds to the free massless case has been seen to be admissible (i.e., provides a unitary dynamics), but the condition itself is not necessary.

We are now in an adequate position to prove that our results about the unitary implementation of the dynamics and the uniqueness of the equivalence class of the representation associated with the massless situation are valid as well for the case of the two-sphere, as we had anticipated. If the spatial manifold is $S^{2}$, equipped with the standard metric, all our assumptions are fulfilled, with eigenvalues given by $\omega_{n}^{2}=n(n+1)$, degeneracy factors equal to $g_{n}=2 n+1$, and symmetry group given by $S O(3)$. Note in particular that $g_{n} / \omega_{n}^{4}$ behaves asymptotically as $1 / n^{3}$, so that the corresponding sequence is indeed summable. Therefore, unitarity and uniqueness of the considered representation are reached for the two-sphere without appealing to the axisymmetry of the field, generalizing in this way the conclusions of Ref. [7] where the axisymmetric restriction was studied.

It is also worth commenting that the summability condition for $g_{n} / \omega_{n}^{4}$ appears to de- 
pend importantly on the dimensionality. Generically, for a compact spatial manifold of dimension $d$, one would expect that the dimension $g_{n}$ of the eigenspaces of the LaplaceBeltrami operator increases for large $n$ as $n^{d-1}$, except if there are suppressions in this degeneracy owing to the specific symmetry of the system. Similarly, one would expect that the eigenvalue $\omega_{n}^{2}$ increases quadratically, as $n^{2}$. All this is actually so for the two-sphere and the three-sphere. Then, the requirement that $g_{n} / \omega_{n}^{4}$ be summable would amount to the summability of the sequence of elements $n^{d-5}$, which is true only in (integer) dimensions equal or smaller than three. These heuristic arguments seem to indicate that, in addition to the compactness of the spatial manifold, the dimensionality plays a fundamental role for the unitary implementation of the dynamics in the Fock representation obtained from the massless field case.

One interesting situation, owing to its application to cosmological perturbations, like in the case of the three-sphere, is when the spatial manifold is the three-torus (equipped with the Euclidean metric). The corresponding eigenvalues of the Laplace-Beltrami operator are labeled by three integers $\left(n_{1}, n_{2}\right.$, and $\left.n_{3}\right)$, one for each dimension, and are given by $\omega_{n}^{2}=n_{1}^{2}+n_{2}^{2}+n_{3}^{2}$. It is possible to check then the summability of the sequence corresponding to elements of the form $g_{n} / \omega_{n}^{4}$. On the other hand, the group of symmetries of the field equation is the product of the three copies of the group of translations in the circle. Note that the representation of this group is not irreducible in the eigenspaces of the Laplace-Beltrami operator. Nonetheless, as we have remarked, this requirement of irreducibility is not a necessary condition to reach the uniqueness of the representation. Given that the representation is irreducible only in the sectors determined by the whole set of indices $\left(n_{1}, n_{2}, n_{3}\right)$, the symplectomorphism $K$ that characterizes the invariant complex structures will now decompose in matricial blocks labeled by $\left(n_{1}, n_{2}, n_{3}\right)$, rather than by $n$. The corresponding $2 \times 2$ matrices $\mathcal{K}_{n_{1} n_{2} n_{3}}$ will now cease to be the same for all the blocks associated with the same eigenspace of the Laplace-Beltrami operator (i.e., they will not depend only on $n$ ). In spite of this, it is possible to show that the proof of Sec. 4 can be generalized taking into account this fact and conclude again the uniqueness of the Fock representation. We will address this issue in more detail elsewhere. 
Finally, let us remark that the proof of uniqueness that we have presented allows one to select a preferred Fock quantization in the list of scenarios and situations discussed in the Introduction of this article. In particular, it is worth noting that the kind of rescaling of the cosmological perturbations by an appropriate time function (determined by the FRW scale factor) which leads to a field equation of the type considered in this work has been frequently employed in the literature to pass to the quantum description of these

perturbations (see e.g. [18], although the discussion there is specialized to the case of a flat FRW background). At least for compact spatial topology, the results of our work demonstrate that the Fock quantization adopted in those cases is in fact the (essentially) unique one which incorporates in a natural way the symmetries of the field equation (as symmetries of the vacuum) and provides a unitary implementation of the quantum dynamics for a fixed classical background.

\section{Acknowledgements}

G.A.M.M. is grateful to Javier Olmedo for suggestions and fruitful discussions. This work was supported by the Spanish MICINN Project FIS2008-06078-C03-03, the Spanish Consolider-Ingenio 2010 Program CPAN (CSD2007-00042), and the DGAPA-UNAM (Mexico) Grant No. IN108309-3.

\section{Appendix A: Calculations for the proof of uniqueness}

In this appendix we will show that inequality (61) suffices to prove the square summability of the sequence $\left\{\sqrt{g_{n}} \lambda_{n} / \kappa_{n}\right\}$, a fact from which one can deduce the unitary equivalence of all the invariant complex structures which implement the dynamics as a unitary transformation.

We will first show that Eq. (61) provides us with a bound on $\sum g_{n}\left|\lambda_{n}\right|^{2} /\left|\kappa_{n}\right|^{2}$. For 
that, we note that

$$
\begin{array}{r}
\int_{E_{\delta}} \sin ^{2}\left[\omega_{n} T+\int_{0}^{T} \mathrm{~d} \bar{t} \frac{f\left(\bar{t}+t_{0}\right)}{2 \omega_{n}}\right] \mathrm{d} T \\
=\int_{\overline{\mathbb{I}}_{L}} \sin ^{2}\left[\omega_{n} T+\int_{0}^{T} \mathrm{~d} \bar{t} \frac{f\left(\bar{t}+t_{0}\right)}{2 \omega_{n}}\right] \mathrm{d} T-\int_{\bar{E}_{\delta}} \sin ^{2}\left[\omega_{n} T+\int_{0}^{T} \mathrm{~d} \bar{t} \frac{f\left(\bar{t}+t_{0}\right)}{2 \omega_{n}}\right] \mathrm{d} T \\
\geq \int_{\overline{\mathbb{I}}_{L}} \sin ^{2}\left[\omega_{n} T+\int_{0}^{T} \mathrm{~d} \bar{t} \frac{f\left(\bar{t}+t_{0}\right)}{2 \omega_{n}}\right] \mathrm{d} T-\delta, \quad \forall n .
\end{array}
$$

We recall that $E_{\delta}$ is the set introduced in the discussion of Luzin's theorem, while $\bar{E}_{\delta}$ is its complement in $\overline{\mathbb{I}}_{L}$, its measure being smaller than $\delta$. In addition, for the integral over $\overline{\mathbb{I}}_{L}$ we find

$$
\begin{array}{r}
\int_{\overline{\mathbb{I}}_{L}} \sin ^{2}\left[\omega_{n} T+\int_{0}^{T} \mathrm{~d} \bar{t} \frac{f\left(\bar{t}+t_{0}\right)}{2 \omega_{n}}\right] \mathrm{d} T=\frac{L}{2}-\frac{\sin \left[2 \omega_{n}(a+L)+\int_{0}^{(a+L)} \mathrm{d} \bar{t} \frac{f\left(\bar{t}+t_{0}\right)}{\omega_{n}}\right]}{4 \omega_{n}+2 f\left(a+L+t_{0}\right) / \omega_{n}} \\
+\frac{\sin \left[2 \omega_{n} a+\int_{0}^{a} \mathrm{~d} \bar{t} \frac{f\left(\bar{t}+t_{0}\right)}{\omega_{n}}\right]}{4 \omega_{n}+2 f\left(a+t_{0}\right) / \omega_{n}}-\frac{1}{8 \omega_{n}^{3}} \int_{\overline{\mathbb{I}}_{L}} \frac{f^{\prime}\left(T+t_{0}\right)}{\left[1+\frac{f\left(T+t_{0}\right)}{2 \omega_{n}^{2}}\right]^{2}} \sin \left[2 \omega_{n} T+\int_{0}^{T} \mathrm{~d} \bar{t} \frac{f\left(\bar{t}+t_{0}\right)}{\omega_{n}}\right] \mathrm{d} T \\
\geq \frac{L}{2}-\frac{\omega_{n}}{\left|4 \omega_{n}^{2}+2 f\left(a+L+t_{0}\right)\right|}-\frac{\omega_{n}}{\left|4 \omega_{n}^{2}+2 f\left(a+t_{0}\right)\right|}-\frac{1}{8 \omega_{n}^{3}} \int_{\overline{\mathbb{I}}_{L}} \frac{\left|f^{\prime}\left(T+t_{0}\right)\right|}{\left[1+\frac{f\left(T+t_{0}\right)}{2 \omega_{n}^{2}}\right]^{2}} \mathrm{~d} T .
\end{array}
$$

In the following, we restrict ourselves to $n \geq n_{0}$, where $n_{0}$ is any fixed (positive) integer such that $\omega_{n_{0}}^{2}$ is larger than the maximum of the function $\left|f\left(T+t_{0}\right)\right| /(2 D)$ in the interval $\overline{\mathbb{I}}_{L}$, and $D<1$ is any fixed constant. Then, given that $\omega_{n}$ increases monotonically with $n$,

$$
\left|1+\frac{f\left(T+t_{0}\right)}{2 \omega_{n}^{2}}\right| \geq 1-\frac{\left|f\left(T+t_{0}\right)\right|}{2 \omega_{n}^{2}} \geq 1-\frac{\left|f\left(T+t_{0}\right)\right|}{2 \omega_{n_{0}}^{2}} \geq 1-D,
$$

for all $T$ in the interval of integration, including its end points. Hence one obtains that, for all $n \geq n_{0}$,

$$
\int_{\overline{\mathbb{I}}_{L}} \sin ^{2}\left[\omega_{n} T+\int_{0}^{T} \mathrm{~d} \bar{t} \frac{f\left(\bar{t}+t_{0}\right)}{2 \omega_{n}}\right] \mathrm{d} T \geq \frac{L}{2}-\frac{1}{2 \omega_{n_{0}}(1-D)}-\frac{\int_{\overline{\mathbb{I}}_{L}}\left|f^{\prime}\left(T+t_{0}\right)\right| \mathrm{d} T}{8 \omega_{n_{0}}^{3}(1-D)^{2}}:=\Lambda_{n_{0}},
$$

where we also assume that $n_{0}$ is such that $\Lambda_{n_{0}}>0$ (this can always be fulfilled with an appropriate choice, since $\Lambda_{n_{0}}$ tends to $L / 2$ when $n_{0}$ tends to infinity).

We now introduce the above result in the last inequality of Eq. (A1). In this way, we get

$$
\int_{E_{\delta}} \sin ^{2}\left[\omega_{n} T+\int_{0}^{T} \mathrm{~d} \bar{t} \frac{f\left(\bar{t}+t_{0}\right)}{2 \omega_{n}}\right] \mathrm{d} T \geq \Lambda_{n_{0}}-\delta
$$


We choose $\delta$ such that $\Lambda_{n_{0}}>\delta$, something which is always possible. Then, it follows from Eq. (61) that, for all $M \geq n_{0}$,

$$
\sum_{n=n_{0}}^{M} g_{n} \frac{\left|\lambda_{n}\right|^{2}}{\left|\kappa_{n}\right|^{2}} \leq \frac{I_{\delta}}{\Lambda_{n_{0}}-\delta}
$$

Since $n_{0}$ is fixed and the above bound is valid for arbitrary large $M$, it follows that the sequence of nonnegative elements $g_{n}\left|\lambda_{n}\right|^{2} /\left|\kappa_{n}\right|^{2}$ is summable, as we wanted to prove.

[1] B. Simon, Topics in Functional Analysis, edited by R.F. Streater (Academic Press, London, UK, 1972).

[2] R.M. Wald, Quantum Field Theory in Curved Spacetime and Black Hole Thermodynamics (Chicago University Press, Chicago, 1994).

[3] A. Corichi, J. Cortez, and H. Quevedo, Ann. Phys. (N.Y.) 313, 446 (2004).

[4] A. Ashtekar and A. Magnon, Proc. R. Soc. Lond. A 346, 375 (1975); A. Ashtekar and A. Magnon-Ashtekar, Pramana 15, 107 (1980).

[5] A. Corichi, J. Cortez, G.A. Mena Marugán, and J.M. Velhinho, Class. Quantum Grav. 23, $6301(2006)$.

[6] A. Corichi, J. Cortez, G.A. Mena Marugán, and J.M. Velhinho, Phys. Rev. D 76, 124031 (2007).

[7] J. Cortez, G.A. Mena Marugán, R. Serôdio, and J.M. Velhinho, Phys. Rev. D 79, 084040 (2009).

[8] J. Cortez, G.A. Mena Marugán, and J.M. Velhinho, Class. Quantum Grav. 25, 105005 (2008).

[9] R.H. Gowdy, Ann. Phys. (N.Y.) 83, 203 (1974).

[10] See also A. Corichi, J. Cortez, and G.A. Mena Marugán, Phys. Rev. D 73, 041502 (2006); Phys. Rev. D 73, 084020 (2006); J. Cortez, G.A. Mena Marugán, and J.M. Velhinho, Phys. Rev. D 75, 084027 (2007). 
[11] J.F. Barbero G., D.G. Vergel, and E.J.S. Villaseñor, Class. Quantum Grav. 25, 085002 (2008).

[12] R.T. Jantzen, J. Math. Phys. 19, 1163 (1978).

[13] E. Lifshitz, Zh. Eksp. Teor. Fiz. 16, 587 (1946); E. Lifshitz and I.M. Khalatnikov, Adv. Phys. 12, 185 (1963).

[14] S.W. Hawking, Astrophys. J. 145, 544 (1966).

[15] D.W. Olson, Phys. Rev. D 14, 327 (1976).

[16] J.M. Bardeen, Phys. Rev. D 22, 1882 (1980).

[17] R. Bradenberger, R. Kahn, and W.H. Press, Phys. Rev. D 28, 1809 (1983).

[18] V. Mukhanov, Physical Foundations of Cosmology (Cambrige University Press, Cambridge, UK, 2005).

[19] V.F. Mukhanov, H.A. Feldman, and R.H. Bradenberger, Phys. Rep. 215, 203 (1992).

[20] J.J. Halliwell and S.W. Hawking, Phys. Rev. D 31, 1777 (1985).

[21] The harmonics for nonzero spatial curvature are described, e.g., in E.R. Harrison, Rev. Mod. Phys. 39, 862 (1967).

[22] U.H. Gerlach and U.K. Sengupta, Phys. Rev. D 18, 1773 (1978).

[23] Handbook of Mathematical Functions, edited by M. Abramowitz and I.A. Stegun, NBS Appl. Math. Ser. Vol. LV (U.S. GPO, Washington, DC, 1970), 9th ed.

[24] I.S. Gradshteyn and I.M. Ryzhik, Table of Integrals, Series, and Products, edited by A. Jeffrey and D. Zwillinger (Academic Press, SanDiego, 2000), 6th edn.

[25] A. Corichi, J. Cortez, and H. Quevedo, Phys. Rev. D 66, 085025 (2002); Class. Quantum Grav. 20, L83 (2003).

[26] R. Honegger and A. Rieckers, J. Math. Phys. 37, 4292 (1996).

[27] D. Shale, Trans. Am. Math. Soc. 103, 149 (1962).

[28] A.A. Kirillov, Elements of the theory of representations (Springer-Verlag, New York, 1976).

[29] A.N. Kolmogorov and S.V. Fomin, Elements of the Theory of Functions and Functional Analysis (Dover, New York, 1999). 